\title{
SMOOTH CONTROL OF A WHEELED OMNIDIRECTIONAL ROBOT
}

\author{
Corrado Guarino Lo Bianco*Aurelio Piazzi * \\ Massimo Romano* \\ * Dipartimento di Ingegneria dell'Informazione \\ Università di Parma - Parco Area delle Scienze 181A \\ I-43100 Parma - Italy \\ CA: phone +390521 905792, fax +390521905723 \\ emailromano@ce.unipr.it
}

\begin{abstract}
The paper investigates and proposes a solution for the smooth control of a wheeled omnidirectional mobile robot. The flexibility of this holonomous robot is fully exploited by means of the control strategy proposed. It is based on two main concepts: a Cartesian path planning and a wheel command generation based on the dynamic inversion of the robot kinematic model. The planned path has to exhibit appropriate geometric characteristics in order to generate wheel command signals with continuous accelerations. In particular, the second order geometric continuity of the path, i.e. the continuity of the curvature, has to be guaranteed. Simulation results concerning a perturbed system are reported.
\end{abstract}

Keywords: Autonomous mobile robots, navigation, trajectory planning, path following, dynamic inversion, smooth control, iterative steering.

\section{INTRODUCTION.}

Wheeled omnidirectional mobile robots (WOMRs) have raised the interest of many researchers because of their intrinsic swiftness in performing autonomous maneuvers. Significant applications of WOMRs have been found in the context of the RoboCup competition (e.g. the Arvand robot (Jamzad, 1999)) and in the unmanned ground vehicles (UGVs) (Moore et al., 2000). Kinematics and dynamics of WOMRs have been recently studied in (Williams et al., 2002) and, for the path-tracking control, an exact feedback linearization approach has been proposed in (Moore et al., 2000).

In this work we propose a novel approach to the path-planning and control of a three wheeled omnidirectional robot. The main features of this approach are:

1 This work was partially supported by MIUR in the context of a Cofin2002 project.
(1) Iterative steering between "extended states" of the WOMRs (Lucibello and Oriolo, 1996; Guarino Lo Bianco and Piazzi, 2002).

(2) Path planning with quintic $\mathrm{G}^{2}$-splines that ensures an overall continuity of the curvature (Piazzi et al., 2002).

(3) Dynamic path inversion procedure to generate control inputs with continuous angular acceleration.

Section 2 introduces the concept of second order geometric continuity of Cartesian paths $\left(\mathrm{G}^{2}-\right.$ paths). Section 3 reports the formal definition of the "extended state" of a WOMR, that is the state vector of the kinematic model augmented with the angular velocities and accelerations of the omnidirectional wheels. Then, a motion planning problem between arbitrary extended states is posed and a preliminary result connecting the posed problem with $\mathrm{G}^{2}$-paths is stated (Proposition 1). A Solution to the motion planning problem is provided by the dynamic path inversion algorithm de- 
scribed in Section 4. A simulation example of the proposed path planning and control of WOMRs is presented in Section 5 for a perturbed model. The last section reports concluding remarks.

\section{NOTATION AND PRELIMINARIES ON $G^{2}$-PATHS}

The Euclidean norm of a vector $\mathbf{p}$ is denoted with $\|\mathbf{p}\|$. Let indicate by $C^{i}$ the set of functions continuous till the $i$-th derivative and by $C_{p}$ the class of piecewise continuous functions. A curve on the $\{x, y\}$-plane can be described by means of the following parametrization $\mathbf{p}(u)$ :

$$
\begin{aligned}
\mathbf{p}:\left[u_{0}, u_{1}\right] & \rightarrow \mathbb{R}^{2} \\
u & \rightarrow[\alpha(u) \beta(u)]^{T}
\end{aligned}
$$

where $\left[u_{0}, u_{1}\right]$ is a real closed interval. The "path" associated to the curve $\mathbf{p}(u)$ is $\mathbf{p}\left(\left[u_{0}, u_{1}\right]\right)$, i.e. the image of $\left[u_{0}, u_{1}\right]$ under the vectorial function $\mathbf{p}(u)$.

Definition 1. A curve $\mathbf{p}(u)$ is regular if $\dot{\mathbf{p}}(u) \in$ $C_{p}\left(\left[u_{0}, u_{1}\right]\right)$ and $\dot{\mathbf{p}}(u) \neq 0 \forall u \in\left[u_{0}, u_{1}\right]$.

The curve length measured along $\mathbf{p}(u)$ is denoted by $s$; it can be expressed as a function $f$ of $u$ as

$$
\begin{aligned}
& f:\left[u_{0}, u_{1}\right] \rightarrow \\
& u \rightarrow s=\int_{u_{0}}^{u} \| \mathbf{p}\left(\xi\left(u_{1}\right)\right] \\
& \mathbf{p}(\xi) \| d \xi
\end{aligned}
$$

Evidently, given a regular curve $\mathbf{p}(u)$, the length function $f(\cdot)$ is continuous over $\left[u_{0}, u_{1}\right]$ and bijective; hence its inverse is continuous too and it will be denoted as

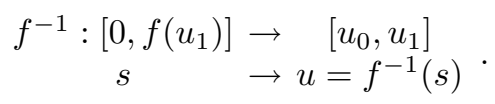

Associated with every point of a regular curve $\mathbf{p}(u)$ there is the orthonormal moving reference system $\{\boldsymbol{\tau}(u), \boldsymbol{\nu}(u)\}$ that is congruent with the axes of the $\{x, y\}$-plane and where $\boldsymbol{\tau}(u)=$ $\dot{\mathbf{p}}(u) /\|\dot{\mathbf{p}}(u)\|$ denotes the unit tangent vector to $\mathbf{p}(u)$.

Definition 2. ( $G^{1}$-curves) A parametric curve $\mathbf{p}(u)$ has first order geometric continuity and we say $\mathbf{p}(u)$ is a $G^{1}$-curve if $\mathbf{p}(u)$ is regular and its unit tangent vector is a continuous function along the curve, i.e. $\boldsymbol{\tau}(\cdot) \in C^{0}\left(\left[u_{0}, u_{1}\right]\right)$

For any regular curve such that $\ddot{\mathbf{p}}(u) \in C_{p}\left(\left[u_{0}, u_{1}\right]\right)$, the scalar curvature can be defined according to the Frenet formula $\dot{\boldsymbol{\tau}}(u)=\kappa_{c}(u) \boldsymbol{\nu}(u)$ (Hsiung, 1997, see p. 109). This defines the curvature function with respect to parameter $u$ :

$$
\begin{array}{cc}
\kappa_{c}:\left[u_{0}, u_{1}\right] & \rightarrow \mathbb{R} \\
u & \rightarrow \kappa_{c}(u) .
\end{array}
$$

According to the theory of planar curves, an explicit expression of $\kappa_{c}(u)$ is

$$
\kappa_{c}(u)=\frac{\dot{\alpha}(u) \ddot{\beta}(u)-\ddot{\alpha}(u) \dot{\beta}(u)}{\left(\dot{\alpha}^{2}(u)+\dot{\beta}^{2}(u)\right)^{3 / 2}} .
$$

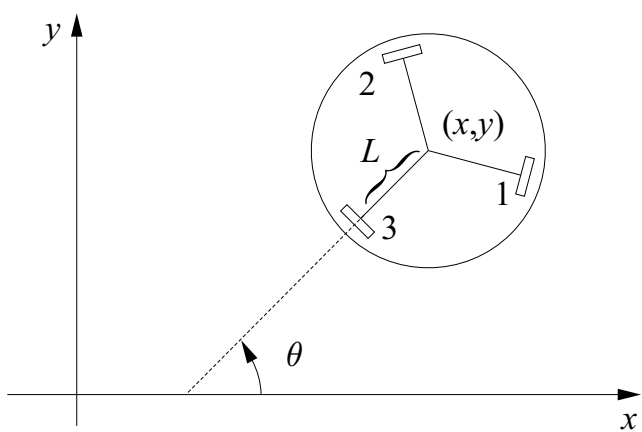

Fig. 1. The WOMR state variables.

The scalar curvature can also be expressed as a function of the curve length $s$. In the following such function will be indicated as

$$
\begin{aligned}
\kappa:\left[0, f\left(u_{1}\right)\right] & \rightarrow \mathbb{R} \\
s & \rightarrow \kappa(s)
\end{aligned}
$$

and the bijectivity of function $f(u)$ makes it possible to write

$$
\kappa(s)=\kappa_{c}\left(f^{-1}(s)\right) .
$$

In view of relation (7), the following result is straightforward.

Lemma 1. For any regular curve $\mathbf{p}(u)$ the curvature function $\kappa_{c}(u)$ is continuous if and only if function $\kappa(s)$ is continuous, i.e. $\kappa_{c}(\cdot) \in C^{0}\left(\left[u_{0}, u_{1}\right]\right)$ $\Leftrightarrow \kappa(\cdot) \in C^{0}\left(\left[0, f\left(u_{1}\right)\right]\right)$.

Definition 3. ( $G^{2}$-curves) A parametric curve $\mathbf{p}(u)$ has second order geometric continuity and we say $\mathbf{p}(u)$ is a $G^{2}$-curve if $\mathbf{p}(u)$ is a $G^{1}$-curve, $\ddot{\mathbf{p}}(\cdot) \in C_{p}\left(\left[u_{0}, u_{1}\right]\right)$ and its scalar curvature is continuous along the curve, i.e. $\kappa_{c}(\cdot) \in C^{0}\left(\left[u_{0}, u_{1}\right]\right)$ or $\kappa(\cdot) \in C^{0}\left(\left[0, f\left(u_{1}\right)\right]\right)$.

$G^{1}$ - and $G^{2}$-curves were originally introduced by Barsky and Beatty (Barsky and Beatty, 1983) in a computer graphics context.

Definition 4. ( $G^{1}$ - and $G^{2}$-paths) A path of a Cartesian space, i.e. a set of points of this space, is a $G^{i}$-path $(i=1,2)$ if there exists a parametric $G^{i}$-curve whose image is the given path.

\section{THE MOTION PLANNING PROBLEM.}

Consider a WOMR whose mobility is obtained by means of three independent wheels displaced as shown in Fig. 1. Each wheel is designed to allow free movements along the transversal axis while traction is guaranteed along the longitudinal axis by means of the actuator motor. A typical wheel for an omnidirectional robot is shown in Fig. 2.

A robot model can be devised by means of kinematic considerations (see e.g. (Kalmár-Nagy et $a l ., 2004))$

$$
\begin{aligned}
& \dot{x}=\frac{r}{3}\left[(\sqrt{3} \cos \theta-\sin \theta) \omega_{1}\right. \\
& \left.\quad-(\sqrt{3} \cos \theta+\sin \theta) \omega_{2}+(2 \sin \theta) \omega_{3}\right] \\
& \dot{y}=\frac{r}{3}\left[(\sqrt{3} \sin \theta+\cos \theta) \omega_{1}\right. \\
& \left.\quad-(\sqrt{3} \sin \theta-\cos \theta) \omega_{2}-(2 \cos \theta) \omega_{3}\right] \\
& \dot{\theta}=\frac{r}{3 L}\left(\omega_{1}+\omega_{2}+\omega_{3}\right)
\end{aligned}
$$




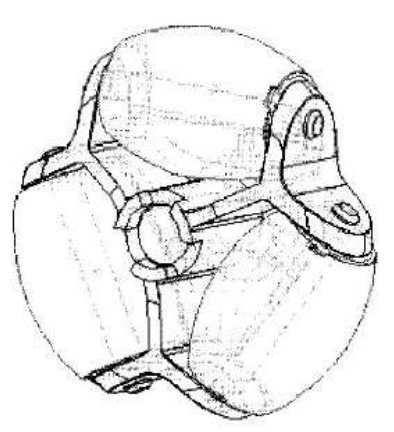

Fig. 2. A typical omnidirectional robot wheel.

where $x, y$ indicate the robot position with respect to a stationary frame, $\theta$ is its heading angle, $L$ is the distance between each wheel and the robot geometrical center, $r$ is the wheels radius, and $\omega_{1}, \omega_{2}$, and $\omega_{3}$ are the angular velocities of the three driving wheels supposed to be positive when generating a counterclockwise movement around the robot spinning axis. These three angular velocities are the robot command signals. Simple dynamic considerations make it possible to assert that the angular velocities of the driving wheels should be continuous with their derivatives, i.e. $\omega_{1}, \omega_{2}, \omega_{3} \in C^{1}$ in order to facilitate the task of the wheel control actuation and to eliminate possible jerky motions of the wheels due to discontinuous accelerations.

From a mathematical standpoint the state of model (8), at time $t$, is given by $\{x(t), y(t), \theta(t)\}$. In the following it is convenient to use an extended state, that includes also the inputs and their derivatives, defined as

$$
\left\{x(t), y(t), \theta(t), \omega_{1}(t), \omega_{2}(t), \omega_{3}(t), \dot{\omega}_{1}(t), \dot{\omega}_{2}(t), \dot{\omega}_{3}(t)\right\} .
$$

Then, the considered motion planning problem can be stated as a reachability problem in the extended state space.

The Problem - Given any assigned travelling time $t_{f}>0$, find the three control inputs $\omega_{1}(t), \omega_{2}(t), \omega_{3}(t) \in C^{1}\left(\left[0, t_{f}\right]\right)$ such that the mobile robot starting from an arbitrary initial extended state

$$
\begin{aligned}
& \mathbf{p}_{A}=\left[\begin{array}{ll}
x_{A} & y_{A}
\end{array}\right]^{T}=[x(0) y(0)]^{T}, \theta_{A}=\theta(0), \\
& \boldsymbol{\omega}_{A}:=\left[\begin{array}{lll}
\omega_{1 A} & \omega_{2 A} & \omega_{3 A}
\end{array}\right]^{T}=\left[\omega_{1}(0) \omega_{2}(0) \omega_{3}(0)\right]^{T} \text {, } \\
& \dot{\omega}_{A}:=\left[\begin{array}{lll}
\dot{\omega}_{1 A} & \dot{\omega}_{2 A} & \dot{\omega}_{3 A}
\end{array}\right]^{T}=\left[\dot{\omega}_{1}(0) \dot{\omega}_{2}(0) \dot{\omega}_{3}(0)\right]^{T} \text {, }
\end{aligned}
$$

reaches the arbitrary final extended state

$$
\begin{gathered}
\mathbf{p}_{B}=\left[\begin{array}{ll}
x_{B} & y_{B}
\end{array}\right]^{T}=\left[x\left(t_{f}\right) y\left(t_{f}\right)\right]^{T}, \theta_{B}=\theta\left(t_{f}\right), \\
\boldsymbol{\omega}_{B}:=\left[\begin{array}{lll}
\omega_{1 B} & \omega_{2 B} & \omega_{3 B}
\end{array}\right]^{T}=\left[\begin{array}{lll}
\omega_{1}\left(t_{f}\right) & \omega_{2}\left(t_{f}\right) \omega_{3}\left(t_{f}\right)
\end{array}\right]^{T}, \\
\dot{\boldsymbol{\omega}}_{A}:=\left[\begin{array}{lll}
\dot{\omega}_{1 B} & \dot{\omega}_{2 B} & \dot{\omega}_{3 B}
\end{array}\right]^{T}=\left[\begin{array}{ll}
\dot{\omega}_{1}\left(t_{f}\right) & \dot{\omega}_{2}\left(t_{f}\right) \dot{\omega}_{3}\left(t_{f}\right)
\end{array}\right]^{T} .
\end{gathered}
$$

Solution to the introduced problem will be provided by the path dynamic inversion procedure described in the next section and the overall motion strategy is then based on an iterative steering approach (Lucibello and Oriolo, 1996). The realtime knowledge of the robot position is used to steer the WOMR from the current extended state to a future extended state in an iterative fashion. In such a way, for the WOMR, swift highperformance motion is possible while intelligent or elaborate robot behaviors are performed.

It is useful to introduce the concept of "nondegenerate" inputs.

Definition 5. Let be given any compact time interval $\mathcal{I} \subset \mathbb{R}$. The inputs $\omega_{1}(t), \omega_{2}(t), \omega_{3}(t)$ defined over $\mathcal{I}$ are said nondegenerate over $\mathcal{I}$ if the equalities $\omega_{1}(t)=\omega_{2}(t)=\omega_{3}(t)$ are never satisfied for any $t \in \mathcal{I}$.

The following result is essential to understand how to plan a desired path connecting $\mathbf{p}_{A}$ with $\mathbf{p}_{B}$.

Proposition 1. Let $t_{f}$ be any given positive real. If a Cartesian path is generated by model (8) with nondegenerate inputs $\omega_{1}(t), \omega_{2}(t), \omega_{3}(t) \in$ $C^{1}\left(\left[0, t_{f}\right]\right)$ then it is a $G^{2}$-path.

Conversely, given any Cartesian $G^{2}$-path there exist nondegenerate inputs $\omega_{1}(t), \omega_{2}(t), \omega_{3}(t) \in$ $C^{1}\left(\left[0, t_{f}\right]\right)$ and initial conditions for model (8) such that the Cartesian path generated by (8) coincides with the given $G^{2}$-path.

Proof of Proposition 1 is omitted for brevity (Guarino Lo Bianco et al., 2004).

\section{THE DYNAMIC PATH INVERSION ALGORITHM.}

A solution for the motion planning problem is proposed in this section. It is mainly based on a dynamic inversion procedure. The aim is to synthesize a feedforward control such that, for any given interval $\left[0, t_{f}\right]$, the WOMR starting at time 0 from the extended state

$$
A=\left\{\mathbf{p}_{A}, \theta_{A}, \boldsymbol{\omega}_{A}, \dot{\boldsymbol{\omega}}_{A}\right\}
$$

will reach, at time $t_{f}$, the extended state

$$
B=\left\{\mathbf{p}_{B}, \theta_{B}, \boldsymbol{\omega}_{B}, \dot{\boldsymbol{\omega}}_{B}\right\}
$$

following a $G^{2}$ Cartesian path joining the assigned points $\mathbf{p}_{A}$ and $\mathbf{p}_{B}$. The degenerate condition must be avoided for any $t \in\left(0, t_{f}\right)$. Note that the degenerate situation is accepted for $t=0$ and for $t=t_{f}$. This is useful, for example, to start or stop the WOMR. Indeed, when $\omega_{1}(t)=\omega_{2}(t)=\omega_{3}(t)$ the linear velocity is equal to zero: this means that the WOMR is still or is turning around its vertical symmetrical axis.

The overall procedure can be divided into four steps. First, the supervisor plans the $G^{2}$-path. This requires to evaluate the interpolating data from the initial and final extended states. Secondly, the desired linear velocity of the WOMR, i.e. $v_{d}(t)$, is synthesized. Then, the angular velocity set-point $\dot{\theta}_{d}(t)$ is planned. Finally, the angular velocities commands $\omega_{1}(t), \omega_{2}(t)$, and $\omega_{3}(t)$ are determined. 
Step 1

The unit tangent vector of the $\mathrm{G}^{2}$-path at point $\mathbf{p}_{A}$ is denoted by $\boldsymbol{\tau}_{A}:=\left[\begin{array}{ll}\tau_{1 A} & \tau_{2 A}\end{array}\right]^{T}$. It can be obtained from the current extended state according to equations

$$
\begin{aligned}
\tau_{1 A}= & \frac{\sqrt{3} \cos \theta_{A}\left(\omega_{1 A}-\omega_{2 A}\right)-\sin \theta_{A}\left(\omega_{1 A}+\omega_{2 A}-2 \omega_{3 A}\right)}{\sqrt{2\left[\left(\omega_{1 A}-\omega_{2 A}\right)^{2}+\left(\omega_{2 A}-\omega_{3 A}\right)^{2}+\left(\omega_{3 A}-\omega_{1 A}\right)^{2}\right]}}, \\
\tau_{2 A}= & \frac{\sqrt{3} \sin \theta_{A}\left(\omega_{1 A}-\omega_{2 A}\right)+\cos \theta_{A}\left(\omega_{1 A}+\omega_{2 A}-2 \omega_{3 A}\right)}{\sqrt{2\left[\left(\omega_{1 A}-\omega_{2 A}\right)^{2}+\left(\omega_{2 A}-\omega_{3 A}\right)^{2}+\left(\omega_{3 A}-\omega_{1 A}\right)^{2}\right]}} .
\end{aligned}
$$

In the same way, the initial path curvature $\kappa_{A}$ can be obtained as

$$
\begin{gathered}
\kappa_{A}=\left\{2 r\left(\omega_{1 A}^{3}+\omega_{3 A}^{3}+\omega_{2 A}^{3}-3 \omega_{1 A} \omega_{2 A} \omega_{3 A}\right)\right. \\
+3 L \sqrt{3}\left[\omega_{1 A}\left(\dot{\omega}_{2 A}-\dot{\omega}_{3 A}\right)\right. \\
\left.\left.+\omega_{3 A}\left(\dot{\omega}_{1 A}-\dot{\omega}_{2 A}\right)+\omega_{2 A}\left(\dot{\omega}_{3 A}-\dot{\omega}_{1 A}\right)\right]\right\} / \\
\quad \quad\left\{\sqrt { 2 } r L \left[\left(\omega_{1 A}-\omega_{2 A}\right)^{2}+\left(\omega_{2 A}-\omega_{3 A}\right)^{2}\right.\right. \\
\left.\left.+\left(\omega_{3 A}-\omega_{1 A}\right)^{2}\right]^{3 / 2}\right\} .
\end{gathered}
$$

In case of degenerate initial conditions, equations (9), (10), and (11) become undefined so that the path boundary conditions must be selected differently. More precisely, if the further condition $\dot{\omega}_{1 A}=\dot{\omega}_{2 A}=\dot{\omega}_{3 A}$ holds both $\tau_{A}$ and $\kappa_{A}$ can be freely chosen. Otherwise, the elements of $\tau_{A}$ have to be evaluated as

$$
\begin{gathered}
\tau_{1 A}=\frac{\sqrt{3} \cos \theta_{A}\left(\dot{\omega}_{1 A}-\dot{\omega}_{2 A}\right)-\sin \theta_{A}\left(\dot{\omega}_{1 A}+\dot{\omega}_{2 A}-2 \dot{\omega}_{3 A}\right)}{\sqrt{2\left[\left(\dot{\omega}_{1 A}-\dot{\omega}_{2 A}\right)^{2}+\left(\dot{\omega}_{2 A}-\dot{\omega}_{3 A}\right)^{2}+\left(\dot{\omega}_{3 A}-\dot{\omega}_{1 A}\right)^{2}\right]}}, \\
\tau_{2 A}=\frac{\sqrt{3} \sin \theta_{A}\left(\dot{\omega}_{1 A}-\dot{\omega}_{2 A}\right)+\cos \theta_{A}\left(\dot{\omega}_{1 A}+\dot{\omega}_{2 A}-2 \dot{\omega}_{3 A}\right)}{\sqrt{2\left[\left(\dot{\omega}_{1 A}-\dot{\omega}_{2 A}\right)^{2}+\left(\dot{\omega}_{2 A}-\dot{\omega}_{3 A}\right)^{2}+\left(\dot{\omega}_{3 A}-\dot{\omega}_{1 A}\right)^{2}\right]}},
\end{gathered}
$$

while $\kappa_{A}$ can be still freely selected.

Analogous equations and considerations can be applied to determine $\boldsymbol{\tau}_{B}:=\left[\begin{array}{ll}\tau_{1 B} & \tau_{2 B}\end{array}\right]^{T}$ and $\kappa_{B}$.

The interpolating data $\mathbf{p}_{A}, \mathbf{p}_{B}, \tau_{1 A}, \tau_{2 A}, \tau_{1 B}, \tau_{2 B}$, $\kappa_{A}, \kappa_{B}$ are then used to plan an appropriate $\mathrm{G}^{2}$ curve $\mathbf{p}(u)$. The interpolating conditions on curve $\mathbf{p}(u)$ are thus

$$
\begin{array}{rlrl}
\mathbf{p}\left(u_{0}\right)=\mathbf{p}_{A}, & \mathbf{p}\left(u_{1}\right)=\mathbf{p}_{B}, \\
\tau_{1}\left(u_{0}\right)=\tau_{1 A}, & & \tau_{2}\left(u_{0}\right)=\tau_{2 A}, \\
\tau_{1}\left(u_{1}\right)=\tau_{1 B}, & & \tau_{2}\left(u_{1}\right)=\tau_{2 B}, \\
\kappa_{c}\left(u_{0}\right)=\kappa_{A}, & & \kappa_{c}\left(u_{1}\right)=\kappa_{B} .
\end{array}
$$

The $G^{2}$-curve $\mathbf{p}(u)$ satisfying the interpolating conditions (14)- (17) can be planned, for example, by means of the $\eta$-splines proposed in (Broggi et al., 1999; Piazzi et al., 2002).

\section{Step 2}

Plan the desired scalar velocity profile $v_{d}(t) \in$ $C^{1}\left(\left[0, t_{f}\right]\right)$ satisfying the end-point boundary conditions and $v_{d}(t) \neq 0, \forall t \in\left(0, t_{f}\right)$. First evaluate the total length of $\mathbf{p}(u)$, i.e. $f\left(u_{1}\right)$, by means of
(2). Obviously, the planned $v_{d}(t)$ must satisfy the condition

$$
\int_{0}^{t_{f}} v_{d}(\xi) d \xi=f\left(u_{1}\right)
$$

The interpolating condition for the velocity profile can be evaluated by taking into account model (8). The initial scalar velocity $v_{A}$ can be derived from the extended state according to the equation

$$
v_{A}=\frac{\sqrt{2} r}{3} \sqrt{\left(\omega_{1 A}-\omega_{2 A}\right)^{2}+\left(\omega_{2 A}-\omega_{3 A}\right)^{2}+\left(\omega_{3 A}-\omega_{1 A}\right)^{2}} .
$$

The scalar acceleration at $\mathbf{p}_{A}$ can be obtained by differentiating the equations of model (8)

$$
\begin{aligned}
\dot{v}_{A}= & \frac{\sqrt{2} r}{3}\left\{\left(\omega_{1 A}-\omega_{2 A}\right)\left(\dot{\omega}_{1 A}-\dot{\omega}_{2 A}\right)\right. \\
& \left.+\left(\omega_{2 A}-\omega_{3 A}\right)\left(\dot{\omega}_{2 A}-\dot{\omega}_{3 A}\right)+\left(\omega_{3 A}-\omega_{1 A}\right)\left(\dot{\omega}_{3 A}-\dot{\omega}_{1 A}\right)\right\} \\
& / \sqrt{\left(\omega_{1 A}-\omega_{2 A}\right)^{2}+\left(\omega_{2 A}-\omega_{3 A}\right)^{2}+\left(\omega_{3 A}-\omega_{1 A}\right)^{2}}
\end{aligned}
$$

Under the hypothesis of initial degenerate condition equation (19) becomes undefined. In this case, the following expression has to be used

$$
\dot{v}_{A}=\frac{\sqrt{2} r}{3} \sqrt{\left(\dot{\omega}_{1 A}-\dot{\omega}_{2 A}\right)^{2}+\left(\dot{\omega}_{2 A}-\dot{\omega}_{3 A}\right)^{2}+\left(\dot{\omega}_{3 A}-\dot{\omega}_{1 A}\right)^{2}} .
$$

Similar equations and considerations can be used to evaluate $v_{B}$ and $\dot{v}_{B}$.

The velocity profile $v(t)$ can be planned according to various schemes. A possible approach is to plan the arc length position $s$ of the WOMR along the $G^{2}$-path at time $t$ as a fifth order polynomial

$$
s_{d}(t)=a_{0}+a_{1} t+a_{2} t^{2}+a_{3} t^{3}+a_{4} t^{4}+a_{5} t^{5},
$$

whose coefficients are selected by imposing (see e.g. (Craig, 1989, p. 237)) the boundary conditions

$$
\begin{array}{cr}
s_{d}(0)=0, & s_{d}\left(t_{f}\right)=f\left(u_{1}\right), \\
\dot{s}_{d}(0)=v_{A}, & \dot{s}_{d}\left(t_{f}\right)=v_{B}, \\
\ddot{s}_{d}(0)=\dot{v}_{A}, & \ddot{s}_{d}\left(t_{f}\right)=\dot{v}_{B} .
\end{array}
$$

The velocity command $v_{d}(t)$ is then synthesized as

$$
v_{d}(t)=\frac{d s_{d}}{d t}(t)
$$

Obviously, in such a way the resulting $v_{d}(t)$ automatically satisfies constraint (18).

The proposed procedure does not guarantee that the degenerate configuration $v_{d}\left(t^{*}\right)=0$ is avoided for any $t^{*} \in\left(0, t_{f}\right)$. If this happens, the supervisor can solve the problem in several ways. For example it can adopt a multi-spline profile (Craig, $1989)$ to ensure the condition $v_{d}(t) \neq 0, \forall t \in\left(0, t_{f}\right)$.

Step 3

The WOMR angular position function $\theta_{d}(t) \in$ $C^{2}\left(\left[0, t_{f}\right]\right)$ has to be selected according to boundary conditions that agree with the initial and final extended states of the robot. Thus, it is first necessary to evaluate appropriate boundary conditions by evaluating the initial and final robot angular velocity and acceleration. The necessary equations can be obtained from (8)

$$
\begin{aligned}
& \dot{\theta}_{A}=\frac{r}{3 L}\left(\omega_{1 A}+\omega_{2 A}+\omega_{3 A}\right), \\
& \dot{\theta}_{B}=\frac{r}{3 L}\left(\omega_{1 B}+\omega_{2 B}+\omega_{3 B}\right), \\
& \ddot{\theta}_{A}=\frac{r}{3 L}\left(\dot{\omega}_{1 A}+\dot{\omega}_{2 A}+\dot{\omega}_{3 A}\right), \\
& \ddot{\theta}_{B}=\frac{r}{3 L}\left(\dot{\omega}_{1 B}+\dot{\omega}_{2 B}+\dot{\omega}_{3 B}\right) .
\end{aligned}
$$


Table 1. Extended states at the interpolating points

\begin{tabular}{c|ccc}
\hline$i \rightarrow$ & $A$ & $P$ & $B$ \\
\hline $\mathbf{p}_{i}$ & {$\left[\begin{array}{l}0 \\
0\end{array}\right]$} & {$\left[\begin{array}{c}1.4253 \\
0.7607\end{array}\right]$} & {$\left[\begin{array}{c}1.2 \\
1.6\end{array}\right]$} \\
$\theta_{i}$ & 0 & 0.0044 & $\pi / 6$ \\
$\boldsymbol{\omega}_{i}$ & {$\left[\begin{array}{l}0 \\
0 \\
0\end{array}\right]$} & {$\left[\begin{array}{c}4.9274 \\
-2.0225 \\
-2.7437\end{array}\right]$} & {$\left[\begin{array}{c}0.45 \\
1.3 \\
0.85 \\
-0.0433 \\
0.3047 \\
-0.2864\end{array}\right]$} \\
$\dot{\boldsymbol{\omega}}_{i}$ & {$\left[\begin{array}{l}0 \\
0 \\
0\end{array}\right]$} & {$\left[\begin{array}{c}0.15 \\
0.4 \\
0.2\end{array}\right]$} \\
\hline
\end{tabular}

The $C^{2}$ continuity requirement can be fulfilled by adopting again a fifth order spline profile

$$
\theta_{d}(t)=b_{0}+b_{1} t+b_{2} t^{2}+b_{3} t^{3}+b_{4} t^{4}+b_{5} t^{5} .
$$

and imposing $(\nu \in \mathbb{Z}$ is freely assignable $)$

$$
\begin{gathered}
\theta_{d}(0):=\theta_{A}, \quad \theta_{d}\left(t_{f}\right):=\theta_{B}+2 \pi \nu, \\
\dot{\theta}_{d}(0):=\dot{\theta}_{A}, \quad \dot{\theta}_{d}\left(t_{f}\right):=\dot{\theta}_{B}, \\
\ddot{\theta}_{d}(0):=\ddot{\theta}_{A}, \quad \ddot{\theta}_{d}\left(t_{f}\right):=\ddot{\theta}_{B} .
\end{gathered}
$$

Note that the desired final orientation $\theta_{B}$ can be reached by spinning several times the robot around its vertical axis.

Step 4

The motion direction is given by

$$
\varphi_{d}(t)=\left.\arg [\boldsymbol{\tau}(u)]\right|_{u=f^{-1}\left(\int_{0}^{t} v_{d}(\xi) d \xi\right)} .
$$

Thus, the desired linear velocity components are

$$
\begin{aligned}
& \dot{x}_{d}(t)=v_{d}(t) \cos \left(\varphi_{d}(t)\right), \\
& \dot{y}_{d}(t)=v_{d}(t) \sin \left(\varphi_{d}(t)\right) .
\end{aligned}
$$

Finally, the wheel command signals can be derived by inverting model (8)

$$
\begin{aligned}
\omega_{1}(t):= & \frac{1}{r} \sin \left[\frac{\pi}{3}-\theta_{d}(t)\right] \dot{x}_{d}(t) \\
& \quad+\frac{1}{r} \cos \left[\frac{\pi}{3}-\theta_{d}(t)\right] \dot{y}_{d}(t)+\frac{L}{r} \dot{\theta}_{d}(t) \\
\omega_{2}(t):=- & \frac{1}{r} \sin \left[\frac{\pi}{3}+\theta_{d}(t)\right] \dot{x}_{d}(t) \\
& +\frac{1}{r} \cos \left[\frac{\pi}{3}+\theta_{d}(t)\right] \dot{y}_{d}(t)+\frac{L}{r} \dot{\theta}_{d}(t) \\
\omega_{3}(t):= & \frac{1}{r} \sin \theta_{d}(t) \dot{x}_{d}(t) \\
& \quad-\frac{1}{r} \cos \theta_{d}(t) \dot{y}_{d}(t)+\frac{L}{r} \dot{\theta}_{d}(t) .
\end{aligned}
$$

The following result highlights the role of the inversion algorithm in steering the WOMR.

Proposition 2. Let us consider any travelling time $t_{f}>0$ and any extended states A and B. Then, the control inputs $\omega_{1}(t), \omega_{2}(t), \omega_{3}(t) \in C^{1}\left(\left[0, t_{f}\right]\right)$, synthesized by the proposed procedure, steer the WOMR from the extended state $\mathrm{A}$, at time 0 , to the extended state $\mathrm{B}$, at time $t_{f}$, in such a way that the generated motion path exactly matches the $G^{2}$-path planned at step 1 of the procedure.

For brevity, the proof of Proposition 2 is omitted (Guarino Lo Bianco et al., 2004).

\section{EXAMPLE WITH A PERTURBED MODEL.}

The control strategy proposed has been tested by means of several simulation experiments. The following parameters have been adopted for the

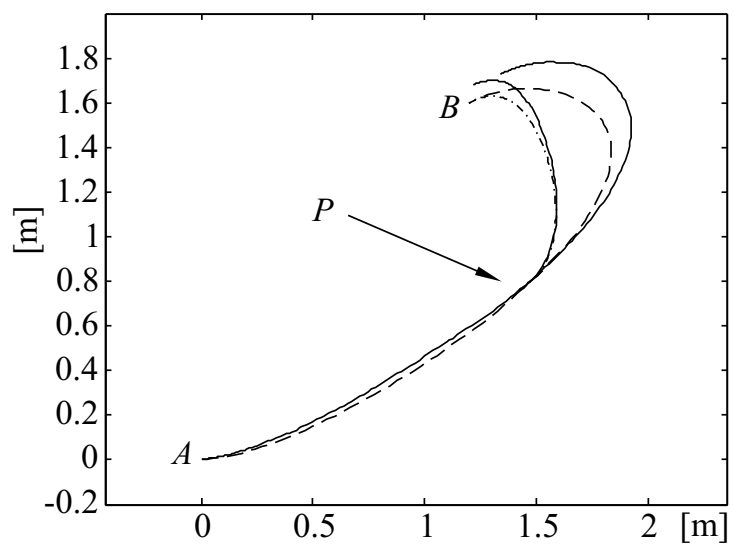

Fig. 3. Originally planned path (dashed line), replanned path (dash-dotted line), and real (solid lines) trajectories. The trajectory is replanned at point $P$.

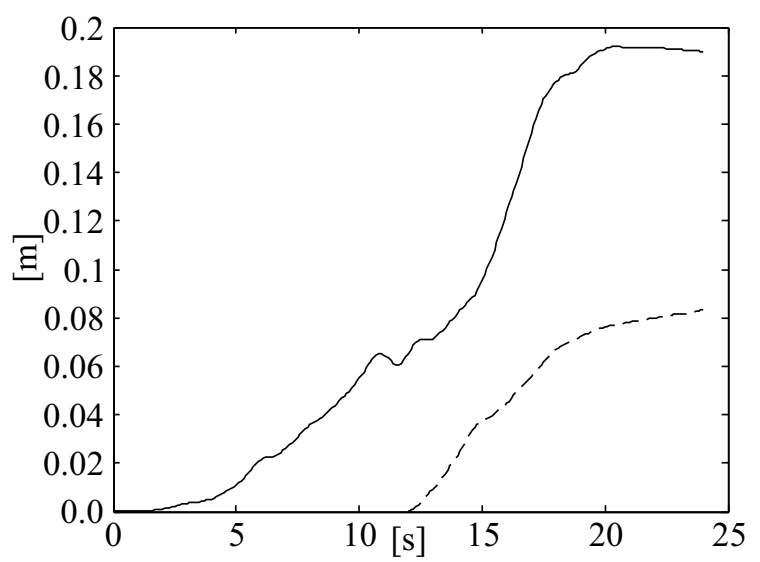

Fig. 4. Distance between planned and real trajectories for the uncertain model. The solid line refers to the first path planning whereas the dashed line refers to the second path planning.

WOMR: $L=0.3 \mathrm{~m}, r=0.05 \mathrm{~m}$. The robot has to move from the extended state $A$ to the extended state $B$ of table 1 with a total travelling time $t_{f}=24 \mathrm{~s}$. A first experiment considers an ideal robot. In this case, a single trajectory has been planned. Thanks to the control strategy proposed in the previous section, the robot follows almost exactly the planned curve. The final position and orientation errors are close to zero and are due to the simulation numerical approximation.

A second pair of simulation experiments consider a more realistic case. The following perturbed model has been considered in order to check the robustness characteristics of the control

$$
\begin{aligned}
& \dot{x}=\frac{r}{3}\left[(\sqrt{3} \cos \theta-\sin \theta) \omega_{1}\right. \\
& \left.\quad-(\sqrt{3} \cos \theta+\sin \theta) \omega_{2}+(2 \sin \theta) \omega_{3}\right]+n_{1}(t) \\
& \dot{y}=\frac{r}{3}\left[(\sqrt{3} \sin \theta+\cos \theta) \omega_{1}\right. \\
& \left.\quad-(\sqrt{3} \sin \theta-\cos \theta) \omega_{2}-(2 \cos \theta) \omega_{3}\right]+n_{2}(t) \\
& \dot{\theta}=\frac{r}{3 L}\left(\omega_{1}+\omega_{2}+\omega_{3}\right)+n_{3}(t)
\end{aligned}
$$

where $n_{1}(t), n_{2}(t)$, and $n_{3}(t)$ are gaussian band limited and rate limited noises. 


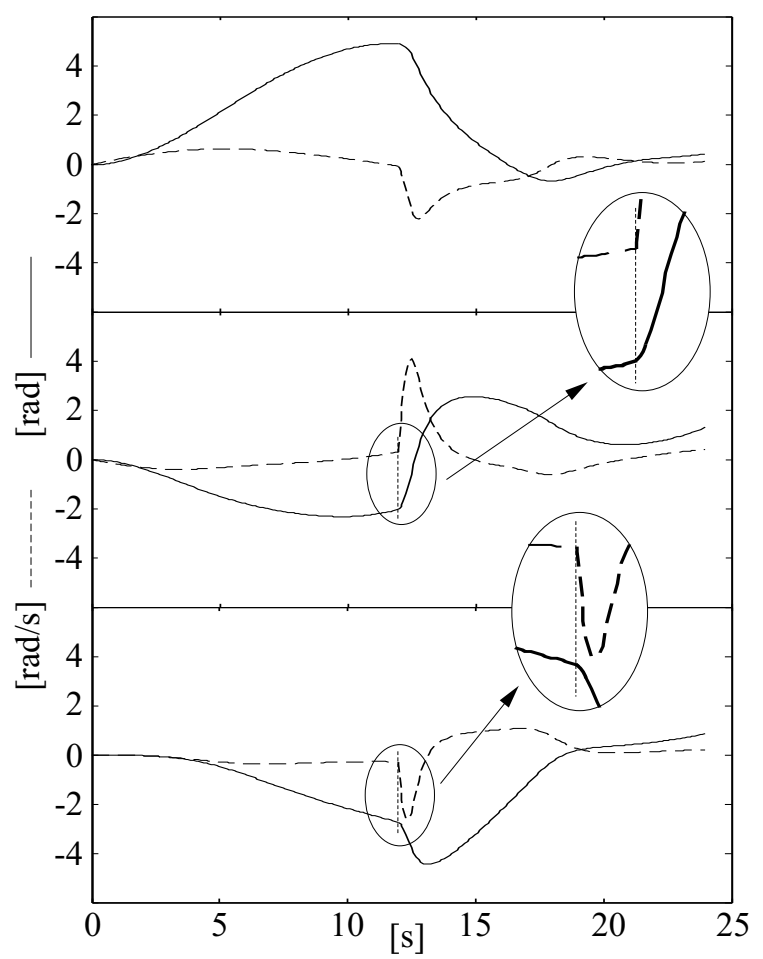

Fig. 5. The three wheels command inputs (solid lines) and their derivatives (dashed lines) for the uncertain model. The details reveal that the three velocities are smoothly joined at the replanning times (i.e $\boldsymbol{\omega} \in C^{1}$ ).

Two different situations are compared in Fig. 3. In the first case the final point $B$ is gained by means of a single planning. Obviously, the robot diverges from the planned path due to the added noise. In the second simulation, at time $t=12 \mathrm{~s}$, corresponding to point $P$ of Fig. 3 , the iterative steering procedure replans the path: the current extend state of the robot (see the $P$ column of table 1 ) is used to generate a new curve such to guarantee the overall $\mathrm{G}^{2}$ continuity of the path. In Fig. 4 the trajectory tracking errors are compared for the two cases. In case of replanning the final displacement error is largely reduced.

Finally, the wheels command inputs and their derivatives are shown in Fig. 5. The continuity of both $\boldsymbol{\omega}$ and $\dot{\boldsymbol{\omega}}$ is guaranteed nevertheless the path replanning occurring at $t=12 \mathrm{~s}$. Moreover, the initial and final $\boldsymbol{\omega}$ and $\dot{\boldsymbol{\omega}}$ coincide with those used for the trajectory planning.

\section{CONCLUSIONS.}

A novel approach for the smooth control of WOMRs has been proposed. It gives the possibility to drive the robot from any extended state to any other with command signals which are continuous with their derivatives. This makes it possible to obtain smooth travelling maneuvers and bounded dynamic solicitations. Joined with an appropriate obstacle avoidance algorithm it could be easily used to navigate inside an hostile environment.

\section{REFERENCES}

Barsky, B. A. and J. C. Beatty (1983). Local control of bias and tension in beta-spline. Computer Graphics 17(3), 193-218.

Broggi, A., M. Bertozzi, A. Fascioli, C. Guarino Lo Bianco and A. Piazzi (1999). The ARGO autonomous vehicle's vision and control systems. International Journal of Intelligent Control and Systems 3(4), 409-441.

Craig, J. J. (1989). Introduction to robotics: mechanics and control. Addison-Wesley. Reading, MA.

Guarino Lo Bianco, C., A. Piazzi and M. Romano (2004). Dynamic path inversion for the motion control of a wheeled omnidirectional robot. Technical report TSC-04/01. Dip. di Ing. dell'Informazione - University of Parma. Italy.

Guarino Lo Bianco, C. and A. Piazzi (2002). Inversion-based control of wheeled mobile robots. In: Proceedings of the IEEE Intelligent Vehicles Symposium. Versailles, France. pp. 190-195.

Hsiung, C.-C. (1997). A First Course in Differential Geometry. International Press. Cambrige, MA.

Jamzad, M. (1999). CS-Sharif ROCS99 team in middle-sized robots league.. Linköping Electronic Articles in Computer and Information Science 4(6), 118-126.

Kalmár-Nagy, T., R. D'Andrea and P. Ganguly (2004). Near-optimal dynamic trajectory generation and control of an omnidirectional vehicle. Robotics and Autonomous Systems 46, 47-64.

Lucibello, P. and G. Oriolo (1996). Stabilization via iterative state steering with application to chained-form systems. In: Proceedings of the 35th IEEE Conference on Decision and Control. Vol. 3. Kobe, Japan. pp. 2614-2619.

Moore, K.L., M. Davidson, V. Bahl, S. Rich and S. Jirgal (2000). Modelling and control of a six-wheeled autonomous robot. In: Proc. of the American Control Conference. Chicago, Illinois. pp. 1483-1490.

Piazzi, A., C. Guarino Lo Bianco, M. Bertozzi, A. Fascioli and A. Broggi (2002). Quintic $G^{2}-$ splines for the iterative steering of visionbased autonomous vehicles. IEEE Transactions on Intelligent Transportations Systems 3(1), 27-36.

Williams, R.L., B.E. Carter, P. Gallina and G. Rosati (2002). Dynamic model with slip for wheeled omnidirectional robots. IEEE Trans. on Robotics and Automation 18(3), 285-293. 